\title{
Multiuser Diversity in Wireless Networks: A Diversity-Multiplexing Perspective
}

\author{
Kaushik Josiam, Dinesh Rajan \\ Department of Electrical Engineering, Southern Methodist University \\ Dallas, TX 75275, USA. Email: \{kjosiam, rajand\}@engr.smu.edu
}

\begin{abstract}
We derive a simple characterization of the diversitymultiplexing (D-MG) tradeoff in three multiuser scenarios - multiple access, broadcast and relay assuming channel knowledge at the transmitters. Specifically, we study the D-MG tradeoff of protocols in literature that opportunistically use the channel knowledge to maximize the information rate. We show that for the multiple access channel with $K$ users, a feedback of $\log K$ bits is sufficient to maximize the multiuser diversity gain. The multiuser diversity gains in all three channels are obtained without explicit cooperation between users.
\end{abstract}

\section{INTRODUCTION}

Three main multiuser scenarios have received significant focus in literature namely multiple access (MAC), broadcast and relay. If the channel is unknown at the transmitters, the optimal D-MG tradeoff has been completely characterized in [1] for a symmetric MAC with no cooperation among users and for various cooperative protocols in multiuser channels in [2]-[5]. With channel state information at transmitter (CSIT), [6] shows that the sum-rate capacity for a MAC can be maximized by opportunistic user selection. Additionally, CSIT was shown to improve the diversity gain due to the multiple users themselves, a gain termed multiuser diversity. In this paper, we characterize the tradeoff between multiuser diversity and multiplexing gains for protocols proposed in [6], [7] for the single antenna MAC and broadcast channels. We also study extensions of opportunistic user selection to the relay channel.

\section{MAin Results}

1) $M A C$ : Consider a fading MAC with $K$ users where the wireless links between the $\mathrm{K}$ users and the receiver are i.i.d. complex Gaussian random variables with zero mean and unit variance. Further, the channels are assumed to be block fading where the channel coefficients may change independently after coherence time, $t_{c}$ symbols. Users communicate based on a time-sharing principle where the user having the maximum instantaneous channel gain accesses the entire bandwidth at any given time [6]. We assume perfect channel state information at the base station, and the user selection is communicated by an instantaneous error-free feedback of $\log K$ bits to all users, every $t_{c}$ symbols. To reduce feedback information, we assume all users transmit at the same power and there is no power control.

Let $r_{i} \in[0,1]$ be the multiplexing gain for the $i^{\text {th }}$ user, achieved using a SNR-indexed code family $\left\{\mathcal{C}_{i}(\mathrm{SNR})\right\}$ such that the data rate scales as $r_{i} \log \mathrm{SNR}$ in the limit SNR $\rightarrow \infty$. In this paper, we focus on the symmetric case, with each user having the same multiplexing gain; hence $r_{i}=\frac{r}{K}$. As SNR $\rightarrow \infty$, the probability of error for each user scales $P_{e}(\mathrm{SNR}) \doteq \mathrm{SNR}^{-d^{*}}$, where $d^{*}$ is the diversity gain.

Theorem 1: Let $K$ users communicate opportunistically on a MAC with per user multiplexing gain given by $r_{1}=\frac{r}{K}$. Then, the achievable diversity by a user, $d^{*}\left(r_{1}\right)$ is defined by the straight line

$$
d^{*}\left(r_{1}\right)=K\left(1-K r_{1}\right)^{+} \text {. }
$$

Compared to the tradeoff for a MAC with no CSIT given in [1], for all rates, $r_{1} \leq 1 /(K+1)$, the MAC with feedback has a diversity order that is strictly greater than the conventional MAC. When the user's multiplexing gain is zero, feedback of $\log K$ bits represents a $K$ fold increase in diversity over conventional MAC scheme.

2) Broadcast Channel: In a broadcast channel, we assume that both the base station and the users have full channel state information. For this case, [7] shows that adopting a similar strategy as the uplink, i.e., transmitting only to the user with the best channel, maximizes the throughput. Using the same argument as the opportunistic MAC, the D-MG tradeoff for a symmetric broadcast channel with CSIT is given in (1).

3) Relay Channel: A relay channel with perfect CSI at all users can be viewed as a concatenation of a MAC followed by a broadcast channel. Consider a protocol where the source transmits to one of the $K$ relays with the best channel at rate $R=r \log$ SNR. The relay in turn, transmits only when its channel to the destination is the best. Thus, the relay buffers information from the source, till it can transmit to the destination. The tradeoff curve $d^{*}(r)$ for a relay channel with $K$ relays with perfect CSI is,

$$
d^{*}(r)=K(1-r)^{+} .
$$

When in addition to the relays, there is a direct link between source and destination, then a simple modification to the proposed relay protocol results in an improvement in the achievable diversity gain. Consider a scheme, where at all odd time instants, the source opportunistically chooses to a transmit to any of the relays or the destination. At even time instants, the destination opportunistically chooses a transmitter from the set which includes all the relays and the source. Then, the D-MG tradeoff $d^{*}(r)$ is given by,

$$
d^{*}(r)=(K+1)(1-r)^{+} \text {. }
$$

\section{REFERENCES}

[1] D. Tse et al., "Diversity-multiplexing tradeoff in multiple-access channels," in IEEE Trans. Inf. Th., vol. 50 no.9, Sep 2004, pp. 1859-1874.

[2] J. Laneman et al., "Disributed space-time-codedprotocols for exploiting cooperative diversit in wireless networks," in IEEE Trans. Inf. Th., vol. 49 no. 10, Oct 2003, pp. 2415-2425.

[3] K. Azarian et al., "On the achievable diversity-multiplexing tradeoff in half-duplex cooperative channels," in IEEE Trans. Inf. Th., vol. 51, no. 12, Dec 2005, pp. 4152-4172.

[4] M. Yuksel and E. Erkip, "Diversity-multiplexing tradeoff in cooperative wireless systems," in Proc. 40th CISS, Princeton, NJ, Mar 2006.

[5] D. Chen and J. Laneman, "The diversity multiplexing tradeoff for the multiaccess relay channel," in Proc. 40th CISS, Princeton, NJ, Mar 2006.

[6] R. Knopp et al., "Information capacity and power control in single-cel multiuser communications," in Proc. IEEE ICC'95, Seattle, WA, June 1995, pp. 331-335.

[7] D. N. Tse, "Optimum power allocation over parallel gaussian broadcast channel," in Proc. ISIT, Ulm, Germany, June 1997, p. 27. 